managed jump load. Inertial measurement units are effective for counting jumps compared to traditional methods, but devices with a minimum threshold may underestimate total load.

Objective To compare jump counts from a commercially-available inertial measurement units to traditional counting methods for male and female youth basketball players.

Design Cross-sectional.

Setting Youth basketball practices and games.

Patients (or Participants) 46 (19 Female) youth basketball players (ages 13-18).

Interventions (or Assessment of Risk Factors) Jump count and height were recorded using an inertial measurement unit (VERT ${ }^{\oplus}$ Classic) and raters counted the number of jumps from a simultaneous video recording.

Main Outcome Measurements The height of VERT ${ }^{\oplus}$ Classic jumps was compared between males and females. The total number of jumps recorded by the VERT ${ }^{\oplus}$ Classic and evaluated by video raters were compared using intraclass correlation coefficient $(\operatorname{ICC}(3, k))$, mean offset, and Bland and Altman limits of agreement.

Results Males (14.1' \pm 3.2 ') had a greater jump height than females (9.9' $\left.\pm 1.3^{\prime}, \mathrm{p}<0.001\right)$. For males, VERT ${ }^{\circledR}$ Classic jumps had excellent reliability with video-counted jumps $(\operatorname{ICC}(3, \mathrm{k})=$ 0.969 ), with a mean offset of -1.9 (fewer VERT ${ }^{\oplus}$ Classic) and limits of agreement -7.1 to 3.2 jumps. For females, VERT ${ }^{\varpi}$ Classic jumps had excellent reliability $(\operatorname{ICC}(3, \mathrm{k})=0.916)$, with a mean offset of -9.3 (fewer VERT ${ }^{\varpi}$ Classic) and limits of agreement -28.2 to 9.6 jumps.

Conclusions The VERT ${ }^{\varpi}$ Classic can provide an estimate of jump load and provides a tool to monitor jump load in relation to overuse knee injuries in youth basketball players. Female youth basketball players appear to perform more jumping movements that are not registered by the VERT ${ }^{\oplus}$ Classic compared to males. Female youth basketball players may need an inertial measurement unit with a lower height threshold for jump count in order to more accurately monitor total jump load.

\section{THE INFLUENCE OF BMI ON CHRONIC INJURIES AND PERFORMANCE IN CLIMBING}

Gudmund Grønhaug. Education, arts and sports, Western Norway University of Applied Sciences, Norway, Sogndal, Norway

\subsection{6/bjsports-2021-IOC.249}

Background Climbing as a weight bearing sport is thought to rely on relative force (force/weight) ratio for performance. As a climbers most valuable tool is the finger flexors, higher BMI is assumed to influence injuries. There is no previous analysis of the influence of BMI on chronic injuries and performance in climbing.

Objective Assess possible associations of BMI on level of performance and injuries in climbing.

Design Retrospective survey.

Setting Item driven web-based questionnaire.

Patients (or Participants) 667 active climbers.

Interventions (or Assessment of Risk Factors) Descriptive statistics, general linear model (GLM) (SPSS V.25 for windows).
Main Outcome Measurements Gender, age, BMI, level of performance, chronic injury past 6 months.

Results 481 male, 186 female, 58.7\% were 26-40 years of age, mean BMI 22.78 (male 22.81 (SD 2.30), female 22.71 (SD 2.21), 76\% intermediate or experienced climbers, 385 reported having chronic injuries. No associations were found for gender $(p=.336)$, level of performance $(p=.840)$, chronic injury $(p=447)$ or BMI.

Conclusions The results from this study suggest that BMI does not influence onset of climbing-related chronic injury or level of performance in climbing.

Clinical relevance The findings in this study suggest that there is no need for undereating to maintain a low BMI to be able to reach elite levels in climbing or to avoid injuries. It seems that muscular growth is according to the resistance in weight bearing sports as well as other sports.

\section{WHY NOT? USE OF HEALTHCARE AND REASONS NOT TO SEEK HEALTHCARE BY NORWEGIAN CLIMBERS WITH CHRONIC INJURIES}

Gudmund Grønhaug, Atle Hole Sæterbakken. Education, arts and sports, Western Norway University of Applied Sciences, Sogndal, Norway

\subsection{6/bjsports-2021-IOC.250}

Background Receiving the right treatment at the right time is vital to avoid sequela or worsening of an injury. Research on use of health care in sports is limited, and reasons for not seeking health care, has to our knowledge, previously not been assessed.

Objective Assess use of healthcare by climbers with chronic injuries using separate analysis on the view on use of healthcare for gender differences and level of performance.

Design Retrospective survey.

Setting Web-based questionnaire.

Patients (or Participants) 385 (289 male, 96 female) active climbers with chronic injuries.

Interventions (or Assessment of Risk Factors) SPSS V.25 for Windows was used to perform descriptive statistics and Pearson's $\chi 2$.

Main Outcome Measurements Gender, use of healthcare (including reasons not to seek healthcare if the patient was not attended by health professionals), level of performance, site of injury, preferred style of climbing.

Results $41.7 \%$ of the female respondents sought healthcare versus $27.3 \%$ of the male respondents $(\chi 2=0.006$, Cramer's V $\phi=0.125)$. The more experienced climbers were less likely to seek healthcare than recreational climbers $(\chi 2=.00$ Cramer's V $\phi=0.207) .70 \%$ self assessed the injury as not serious enough to seek health care. $60 \%$ said that they did not think a health professional could help with the injury.

Conclusions Use of healthcare amongst climbers with a chronic injury is limited and injured climbers self-assess the injury before seeking medical aid. Experience is a strong predictor for not seeking healthcare after an injury. Some of those who do not seek healthcare after self-assessing the injury might be underestimating the seriousness of the injury due to lack of confidence in the health professionals' abilities to help. 